\section{Farbstofflaser befreit von „Hornzipfeln“}

\begin{abstract}
Hirsuties papillaris penis besitzen keinen Krankheitswert und bedürfen daher auch keiner Behandlung. Wird ihre Entfernung trotzdem gewünscht, scheint der gepulste Farbstofflaser (PDL) eine effektive und nebenwirkungsarme Methode darzustellen, die störenden Gebilde loszuwerden.
\end{abstract}

$E_{w}^{\text {to }}$ wa $15 \%$ aller Männer haben sie: Weißliche bis rote, papilläre oder filiforme Knötchen, die den proximalen Rand der Glans penis reihenförmig besetzen. Bei den Hirsuties papillaris penis („Hornzipfeln“) handelt es sich um atavistische Fehlbildungen, die keinen Krankheitswert haben und keine Beschwerden verursachen. Dennoch wurden über die Zeit diverse Verfahren eingesetzt, um ihr Erscheinungsbild zu mindern oder zum Verschwinden zu bringen - meistens mit gemischtem Erfolg. So können Kürettage und Kryotherapie mit flüssigem Stickstoff zwar die Läsionen beseitigen, dafür aber unschöne Narben hinterlassen. Mit dem $\mathrm{CO}_{2}$-Laser werden zwar kosmetisch befriedigende Ergebnisse erzielt, die Hautverletzungen erhöhen aber das Infektionsrisiko und erfordern eine intensive Nachsorge. Als Erfolg versprechen- de und schonende Alternative empfehlen kanadische Dermatologen nun den gepulsten Farbstoff-Laser (PDL).

„Der gepulste Farbstofflaser bietet Dermatologen eine neue Behandlungsoption für Hirsuties papillaris penis, die sicher und einfach durchzuführen ist und zu hervorragenden ästhetischen Ergebnissen führt", sind Priya Sapra und Kollegen vom Institute of Cosmetic and Laser Surgery in Oakville überzeugt. Ihr Urteil stützt sich auf die Behandlung von vier Patienten, über deren Verlauf sie in "JAMA Dermatology“ berichten.

Eine Stunde vor der Lasertherapie wurde ein Lokalanästhetikum auf die Eichel aufgetragen. Die Behandlung mit dem 595-nm-PDL (6-10 J/cm2, 0,5 ms, $5 \mathrm{~mm}$ ) wurde von den Patienten als nicht oder nur leicht unangenehm beschrieben. Bei allen war nach dem Lasern eine leichte Rötung oder Ergussbildung zu beobachten. Die Patienten waren aufgefordert, fünf Tage eine antibiotische Creme aufzutragen. Bei keinem kam es zu Infektionen, Narbenbildung oder anderen Komplikationen.

Ein Patient war bereits nach der ersten Sitzung mit der erzielten signifikanten Reduktion der Papeln zufrieden, sodass nicht noch einmal gelasert wurde. Bei weiteren zwei Patienten konnten mit insgesamt zwei und bei dem verbleibenden Patienten mit insgesamt drei Sitzungen die Knötchen vollständig zum Verschwinden gebracht werden.

Fazit: „Die Fallberichte zeigen die Überlegenheit des PDL gegenüber anderen Strategien bei Hirsuties papillaris penis", schreiben Sapra et al. Neben der guten Verträglichkeit und der Sicherheit betonen sie das „herausragende kosmetische Ergebnis, das mit wenigen Sitzungen erreicht wird".

Dr. Beate Schumacher

Sapra P et al. Pearly Penile Papules. Effective Therapy With Pulsed Dye Laser. JAMA Dermatol 2013 Apr 17; Epub ahead of print

\section{Atopische Dermatitis: Erwachsene unzureichend therapiert}

\section{Die Versorgung von erwachsenen Patienten mit atopischer Dermatitis zeigt klare Defizite. Zu diesem Schluss kommt eine Querschnittstudie der Universität Hamburg. Knapp ein Drittel der Betroffenen leidet unter nächtlichem Juckreiz.}

n der Studie AtopicHealth werteten die Autoren deutschlandweit 1.678 Fragebögen-Sets von Ärzten und Patienten aus 91 dermatologischen Praxen aus. Die im Mittel 38-jährigen Patienten litten durchschnittlich bereits seit $22 \mathrm{Jah}-$ ren an ihrer Hauterkrankung. Am häufigsten waren die Handinnenflächen sowie die Innenseiten der Arme betroffen.

Die gesundheitsbezogene Lebensqualität auf einer Skala von 0-100 (EQ-5dVAS, 100 als maximale Lebensqualität) betrug 63,6; der Dermatologie-Lebensqualitätsindex zwischen 0-30 Punkten (DLQI 0-30, 30 als minimale Lebens- qualität) lag bei 8,5. Etwa ein Drittel der Patienten verspürte deutliche Einbußen an Lebensqualität durch die Erkrankung.

$26,6 \%$ der Studienteilnehmer berichteten, sie litten wegen des starken Juckreizes oft oder gar jede Nacht unter Schlaflosigkeit. 35,8\% gaben an, sie würden dabei häufig oder immer kratzen, teilweise bis zum Auftreten von Hautblutungen. Als Auslöser für den Juckreiz oder die Verschlechterung des Hautbildes machten rund $73 \%$ der Patienten ein emotionales Ereignis oder eine Stresssituation verantwortlich.
90,4\% der Atopiker hatten in den vergangenen fünf Jahren rückfettende Substanzen verwendet, $85,5 \%$ topische Kortikosteroide. $42,5 \%$ wurden mindestens einmal mit einem topischen Calcineurininhibitor behandelt. Die Therapien erzielten bei $75,8 \%$ der Patienten jedoch nur mäßigen oder gar keinen Erfolg.

Fazit: Die Studienergebnisse sprechen für eine klare Unterversorgung der $\mathrm{Pa}$ tienten. Die Autoren plädieren für eine noch bessere Umsetzung der Empfehlungen. Zudem sollten Probleme, die im Rahmen der Behandlung auftreten und den Behandlungserfolg stören, systematisch analysiert werden.

Dr. Christine Starostzik

Langenbruch A et al. Quality of health care of atopic eczema in Germany: results of the national health care study AtopicHealth. J Eur Acad Dermatol Venereol 2013 Apr 5; Epub ahead of print 